\title{
THE DISTRIBUTION OF PATH LENGTHS ON DIRECTED WEIGHTED GRAPHS
}

\author{
AVNER KIRO, YOTAM SMILANSKY, UZY SMILANSKY
}

\begin{abstract}
We consider directed weighted graphs and define various families of path counting functions. Our main results are explicit formulas for the main term of the asymptotic growth rate of these counting functions, under some irrationality assumptions on the lengths of all closed orbits on the graph. In addition we assign transition probabilities to such graphs and compute statistics of the corresponding random walks. Some examples and applications are reviewed.
\end{abstract}

\section{Introduction and Main Results}

Questions regarding the distribution of path lengths on directed weighted graphs are encountered in various fields of study of mathematics and physics. They arise naturally in dynamics and the study of closed orbits of suspensions of shifts of finite type, see among others [PP1], [PP2], [Gu and [CT], and the more recent $[\mathrm{KS}$ and $\mathrm{BPP}$.

Our motivations for counting paths on weighted graphs are diverse. The second author's motivation originates in the study of a model of mathematical quasicrystals which we call multiscale substitution tilings, and in the study of equidistribution of what is known as Kakutani's partitions, first described in $\mathrm{Ka}$. The connection to problems concerning path counting on weighted graphs is introduced in subsection 5.2 The third author's motivation is rooted in theoretical physics, specifically in the spectral properties of the Schrödinger operator for systems which are chaotic in the classical limit and for metric graphs GS. Of particular relevance are studies of the distribution of delay (transit) times through chaotic scatterers such as e.g., the scattering of ultra-short electromagnetic pulses by complex molecules, or traversing networks of transmission lines $\mathrm{An}]$ and $\mathrm{SmU}$.

1.1. Counting paths in graphs. Let $G=(\mathcal{V}, \mathcal{E}, l)$ be a directed weighted metric graph with a set $\mathcal{V}=\{1, \ldots, n\}$ of vertices and a set $\mathcal{E}$ of edges. A positive weight is assigned to each edge $\alpha \in \mathcal{E}$, and we think of this weight as the length of $\alpha$. For a path $\gamma$ connecting two vertices in $\mathcal{V}$, the path metric $l$ is defined to be the sum of the weights of the edges in $\gamma$. When considering paths which do not necessarily terminate at a vertex of $G$, the path metric is defined by $l(\gamma)=a$ if the path $\gamma$ is isometric to $[0, a] \subset \mathbb{R}$. Throughout this paper $G$ is assumed to be a strongly connected multigraph, that is a graph which admits a path from every vertex $i \in \mathcal{V}$ to every vertex $j \in \mathcal{V}$, and loops and multiple edges are allowed.

We say that $G$ is a graph of incommensurable orbits, or incommensurable for short, if there exist at least two closed paths in $G$ of lengths $a, b$ such that $a \notin \mathbb{Q} b$. This irrationality condition on the lengths of the edges is equivalent to the set of lengths of all closed orbits in $G$ not being a uniformly discrete subset of $\mathbb{R}$. Indeed, if there exist two closed paths in $G$ of lengths $a, b$ such that $a \notin \mathbb{Q} b$, then by Dirichlet's approximation theorem for every $\varepsilon>0$ there exist $p, q \in \mathbb{N}$ such that $|a q-p b|<\varepsilon$, and so the set of lengths of closed orbits in $G$ is not uniformly discrete. Conversely, if the set of lengths of closed orbits is rationally dependent, then the finiteness of the graph implies that there is a finite set $L$ of lengths for which the length of any closed orbit in $G$ is a linear combination with integer coefficients of elements in $L$. It follows that the set of lengths of closed orbits in $G$ is uniformly discrete.

Let $i, j \in \mathcal{V}$ be a pair of vertices in $G$, and assume that there are $k \geq 0$ edges $\alpha_{1}, \ldots, \alpha_{k}$ from $i$ to $j$. The matrix valued function $M: \mathbb{C} \rightarrow M_{n}(\mathbb{C})$, which we call the graph matrix function of $G$, is defined by

$$
M_{i j}(s)=e^{-s \cdot l\left(\alpha_{1}\right)}+\cdots+e^{-s \cdot l\left(\alpha_{k}\right)}
$$

and $M_{i j}(s)=0$ if $i$ is not connected to $j$ by an edge. Note that the restriction of $M$ to $\mathbb{R}$ is real valued.

Theorem 1. Let $G$ be a strongly connected incommensurable graph. There exist a positive constant $\lambda$ and a matrix $Q \in M_{n}(\mathbb{R})$ with positive entries such that 
(i) The number of paths from $i \in \mathcal{V}$ to $j \in \mathcal{V}$ of length at most $x$ grows as

$$
\frac{1}{\lambda} Q_{i j} e^{\lambda x}+o\left(e^{\lambda x}\right), \quad x \rightarrow \infty .
$$

(ii) Let $\alpha \in \mathcal{E}$ be an edge in $G$ which originates in vertex $j \in \mathcal{V}$. The number of paths of length exactly $x$ from some vertex $i$ to a point on the edge $\alpha$ grows as

$$
\frac{1-e^{-l(\alpha) \lambda}}{\lambda} Q_{i j} e^{\lambda x}+o\left(e^{\lambda x}\right), \quad x \rightarrow \infty .
$$

The constant $\lambda$ is the maximal real value for which the spectral radius of $M$ is equal to 1 , and

$$
Q=Q(M(\lambda))=\frac{\operatorname{adj}(I-M(\lambda))}{-\operatorname{tr}\left(\operatorname{adj}(I-M(\lambda)) \cdot M^{\prime}(\lambda)\right)}
$$

where $M^{\prime}$ is the entry-wise derivative of $M$, and $\operatorname{adj} A$ is the adjugate or classical adjoint matrix of $A$, that is the transpose of its cofactor matrix.

Example. Let $G$ be the directed weighted graph which appears in Figure 1 .

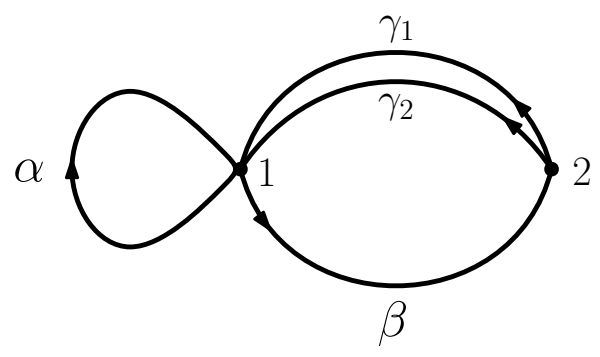

Figure 1. Graph with two vertices $\mathcal{V}=\{1,2\}$ and four edges $\mathcal{E}=\left\{\alpha, \beta, \gamma_{1}, \gamma_{2}\right\}$.

The graph matrix function of $G$ is given by

$$
M(s)=\left(\begin{array}{cc}
e^{-l(\alpha) s} & e^{-l(\beta) s} \\
e^{-l\left(\gamma_{1}\right) s}+e^{-l\left(\gamma_{2}\right) s} & 0
\end{array}\right) .
$$

Putting for example

$$
\begin{array}{ll}
l(\alpha)=\log 2, & l\left(\gamma_{1}\right)=\log \frac{3}{2}, \\
l(\beta)=\log 2, & l\left(\gamma_{2}\right)=\log 3,
\end{array}
$$

we get $\lambda=1$ and

$$
Q=\frac{6}{\log 432}\left(\begin{array}{cc}
1 & \frac{1}{2} \\
1 & \frac{1}{2}
\end{array}\right),
$$

and so by the second part of Theorem 1 , the number of paths of length exactly $x$ from vertex 1 to a point on the edge $\gamma_{2}$ grows as

$$
\frac{1-e^{-l\left(\gamma_{2}\right) \lambda}}{\lambda} Q_{12} e^{\lambda x}+o\left(e^{\lambda x}\right)=\frac{e^{x}}{\log \sqrt{432}}+o\left(e^{x}\right), \quad x \rightarrow \infty .
$$

1.2. Weighted random walks on graphs. Let $\alpha \in \mathcal{E}$ be an edge which originates at $i \in \mathcal{V}$. Denote by $p_{i \alpha}>0$ the probability that a walker who is passing through vertex $i$ chooses to continue his walk through edge $\alpha$, and assume that the sum of the probabilities over all edges originating at a given vertex is less than or equal to 1 , for all vertices in $G$. Let $\alpha_{1}, \ldots, \alpha_{k}$ be the edges connecting vertex $i$ to vertex $j$. The graph probability matrix function $N: \mathbb{C} \rightarrow M_{n}(\mathbb{C})$ is defined by

$$
N_{i j}(s)=p_{i \alpha_{1}} e^{-s \cdot l\left(\alpha_{1}\right)}+\cdots+p_{i \alpha_{k}} e^{-s \cdot l\left(\alpha_{k}\right)}
$$

and $N_{i j}(s)=0$ if $i$ is not connected to $j$ by an edge. Note that the restriction of $N$ to $\mathbb{R}$ is real valued. If the sum of the probabilities over all edges originating at a given vertex is strictly less than 1 , there is a positive probability that the walker does not choose any of the edges and instead leaves the graph. 
Theorem 2. Let $G$ be a strongly connected incommensurable graph, and consider a walker on $G$ advancing at constant speed 1 . There exist a non-positive constant $\lambda$ and a matrix $Q \in M_{n}(\mathbb{R})$ with positive entries such that

(ii) ${ }^{1}$ Let $\alpha \in \mathcal{E}$ be an edge in $G$ which originates in vertex $j \in \mathcal{V}$. The probability that a walker who has left some vertex $i \in \mathcal{V}$ at time $t=0$ is on the edge $\alpha \in \mathcal{E}$ at time $t=T$, where $\alpha$ originates at $j$ and has probability $p_{j \alpha}$, decays as

$$
p_{j \alpha} \frac{1-e^{-l(\alpha) \lambda}}{\lambda} Q_{i j} e^{\lambda T}+o\left(e^{\lambda T}\right), \quad T \rightarrow \infty
$$

whenever $\lambda<0$. In the case $\lambda=0$, the probability tends to

$$
p_{j \alpha} l(\alpha) Q_{i j}, \quad T \rightarrow \infty .
$$

As in the previous theorem, the constant $\lambda$ is the maximal real value for which the spectral radius of $N$ is equal to 1 , and

$$
Q=Q(N(\lambda))=\frac{\operatorname{adj}(I-N(\lambda))}{-\operatorname{tr}\left(\operatorname{adj}(I-N(\lambda)) \cdot N^{\prime}(\lambda)\right)} .
$$

As a direct corollary we have

Corollary 1. In the settings of the previous theorem, denote by $\mathcal{E}(j)$ the set of edges in $G$ with origin at vertex $j \in \mathcal{V}$. The probability that a walker who has left vertex $i \in \mathcal{V}$ at time zero is still on the graph $G$ at time $T$ decays as

$$
\sum_{j \in \mathcal{V}} \sum_{\alpha \in \mathcal{E}(j)} p_{j \alpha} \frac{1-e^{-l(\alpha) \lambda}}{\lambda} Q_{i j} e^{\lambda T}+o\left(e^{\lambda T}\right), \quad T \rightarrow \infty
$$

whenever $\lambda<0$.

Remark 1. It will follow from Remark 4 that in the case $\lambda=0$ the matrix $N(0)$ is right stochastic and that the probability described in the previous corollary is 1 .

The random walk defined above can be generalized by considering a random walk on the edges, and the transition probability $p_{\beta, \alpha}$ from an edge $\alpha$ to an edge $\beta$ vanishes unless $\beta$ originates at the vertex where $\alpha$ terminates. Let $d$ be the number of directed edges on the graph. The graph probability matrix function $W: \mathbb{C} \rightarrow M_{d}(\mathbb{C})$ is defined by

$$
W_{\beta, \alpha}(s)=p_{\beta, \alpha} e^{-s \cdot l(\alpha)} .
$$

The analogue of Theorem 2 and its Corollary 1 in the present case follow directly from the discussion above.

1.3. Acknowledgments. We would like to thank Anton Malyshev, Mikhail Sodin, Yaar Solomon and Barak Weiss for their valuable contribution to this work. In addition we thank Thomas Jordan, Zemer Kosloff, Jens Marklof, Frédéric Paulin, Omri Sarig, Richard Sharp and David Simmons for helpful remarks regarding relevant results. We are thankful to the referee for several remarks and suggestions which helped us to improve this work. The first author is partially supported by the Israel Science Foundation, grant 382/15 and by the United States - Israel Binational Science Foundation, grant 2012037. The second author is supported by the Israel Science Foundation, grant 2095/15.

\section{Matrices, the Theory of Perron-Frobenius and Graphs}

A real valued matrix $A \in M_{n}(\mathbb{R})$ is called positive if all entries of $A$ are positive and non-negative if all entries of $A$ are non-negative. $A$ is called primitive if there exists $k \in \mathbb{N}$ for which $A^{k}$ is positive and irreducible if for every pair of indices $i, j$ there exists $k \in \mathbb{N}$ for which $\left(A^{k}\right)_{i j}>0$.

\footnotetext{
${ }^{1}$ Previous versions of this paper included a result that appeared as part $(i)$ of Theorem 2 . We have found a mistake in the formulation of this result, and it is therefore omitted from this version.
} 
2.1. The Perron-Frobenius Theorem. The following results are due to Perron and Frobenius (full statements and proofs can be found in chapter XIII of [Ga]).

Theorem. Let $A \in M_{n}(\mathbb{R})$ be a non-negative and irreducible matrix.

1. There exists $\mu>0$ which is a simple eigenvalue of $A$, and $\left|\mu_{j}\right| \leq \mu$ for any other eigenvalue $\mu_{j}$. We call $\mu$ the Perron-Frobenius eigenvalue.

2. There exist $v, u \in \mathbb{R}^{n}$ with positive entries such that $A v=\mu v$ and $u^{T} A=\mu u^{T}$. Moreover every right eigenvector with non-negative entries must be a positive multiple of $v$ (similarly for left eigenvectors and $u)$.

Theorem. Let $A \in M_{n}(\mathbb{R})$ be a primitive matrix.

1. There exists $\mu>0$ which is a simple eigenvalue of $A$, and $\left|\mu_{j}\right|<\mu$ for any other eigenvalue $\mu_{j}$. We call $\mu$ the Perron-Frobenius eigenvalue.

2. There exist $v, u \in \mathbb{R}^{n}$ with positive entries such that $A v=\mu v$ and $u^{T} A=\mu u^{T}$. Moreover every right eigenvector with non-negative entries must be a positive multiple of $v$ (similarly for left eigenvectors and $u)$.

3. The following limit holds

$$
\lim _{k \rightarrow \infty}\left(\frac{1}{\mu} A\right)^{k}=\frac{v u^{T}}{u^{T} v} .
$$

The limit matrix $P=\frac{v u^{T}}{u^{T} v}$ is called the Perron projection of $A$.

2.2. Perron's projection. Given an irreducible matrix $A$, there are additional ways to represent its Perron projection $P$, as shown bellow

Lemma 1. Let $A$ be an irreducible matrix with Perron-Frobenius eigenvalue $\mu$ and a Perron projection P. Then

$$
P=\frac{\operatorname{adj}(\mu I-A)}{\operatorname{tr}(\operatorname{adj}(\mu I-A))}
$$

Proof. Let $v$ and $u$ be eigenvectors as in the Perron-Frobenius theorem. The columns of $P$ are scalar multiples of $v$, and the rows of $P$ are scalar multiples of $u^{T}$, and so the column space of $P$ is spanned by $v$ and the row space by $u$. Denote by $V$ the subspace of $M_{n}(\mathbb{R})$ consisting of matrices with these row and column spaces, and notice that $\operatorname{dim} V=1$. Since $\mu$ is an eigenvalue of $A$ we have

$$
(\mu I-A) \cdot \operatorname{adj}(\mu I-A)=\operatorname{det}(\mu I-A) I=0,
$$

and so every column of $\operatorname{adj}(\mu I-A)$ is an eigenvector of $A$ corresponding to $\mu$. By the Perron-Frobenius theorem all columns of adj $(\mu I-A)$ must be scalar multiples of $v$ and so the column space of adj $(\mu I-A)$ is spanned by $v$. Similarly, using adj $(\mu I-A) \cdot(\mu I-A)=0$ we deduce that the row space of adj $(\mu I-A)$ is spanned by $u$, and so $\operatorname{adj}(\mu I-A) \in V$. Since $V$ is one dimensional adj $(\mu I-A)=\alpha P$ for some $\alpha \in \mathbb{R}$. Next, since $P v=v$, and $P w=0$ for every $w \in(\operatorname{span}\{u\})^{\perp}$, the Perron projection $P$ is similar to the matrix $\operatorname{diag}(1,0, \ldots, 0)$. Therefore $\operatorname{tr} P=1$, and so

$$
\operatorname{tr}(\operatorname{adj}(\mu I-A))=\operatorname{tr}(\alpha P)=\alpha \operatorname{tr} P=\alpha,
$$

finishing the proof.

Corollary 2. Let $p_{A}$ be the characteristic polynomial of $A$, then

$$
P=\frac{\operatorname{adj}(\mu I-A)}{\left.\frac{d}{d x} p_{A}(x)\right|_{x=\mu}} .
$$

Proof. Jacobi's formula for the derivative of the determinant of a matrix is given by

$$
\frac{d}{d x} \operatorname{det} B(x)=\operatorname{tr}\left(\operatorname{adj}(B(x)) B^{\prime}(x)\right)
$$

and so using this formula, the corollary follows from the previous lemma for $B(x)=x I-A$.

Remark 2. This result and others concerning the theory of Perron-Frobenius may be found in [Se]. Another proof for Corollary 2 can be derived by direct computation using the following identity

$$
\operatorname{adj}(\lambda I-A)=A^{n-1}+\left(\lambda+p_{n-1}\right) A^{n-2}+\cdots+\left(\lambda^{n-1}+p_{n-1} \lambda^{n-2}+\cdots+p_{1}\right) I
$$


where $p_{A}(x)=x^{n}+p_{n-1} x^{n-1}+\cdots+p_{0}$ and $\lambda \in \mathbb{R}$ (see [Ga] or [FDC]). Let $\mu$ be the Perron-Frobenius eigenvalue and $v$ a corresponding eigenvector, we compute

$$
\begin{aligned}
\operatorname{adj}(\mu I-A) v & =\left[\mu^{n-1}+\left(\mu+p_{n-1}\right) \mu^{n-2}+\cdots+\left(\mu^{n-1}+p_{n-1} \mu^{n-2}+\cdots+p_{1}\right)\right] v \\
& =\left[n \mu^{n-1}+(n-1) p_{n-2} \mu^{n-3}+\cdots+p_{1}\right] v \\
& =p_{A}^{\prime}(\mu) v .
\end{aligned}
$$

Recall that $\operatorname{adj}(\mu I-A)=\alpha P$ for some $\alpha \in \mathbb{R}$. Since

$$
p_{A}^{\prime}(\mu) v=\operatorname{adj}(\mu I-A) v=\alpha P v=\alpha v,
$$

it follows that $\alpha=\operatorname{tr}(\operatorname{adj}(\mu I-A))=p_{A}^{\prime}(\mu)$.

Corollary 3. Let $\left(\mu, \mu_{2}, \ldots, \mu_{n}\right)$ be the eigenvalues of $A$, perhaps with repetitions. Then

$$
P=\frac{\prod\left(A-\mu_{i} I\right)}{\prod\left(\mu-\mu_{i}\right)} .
$$

Proof. Using the representation of $\operatorname{adj}(\mu I-A)$ as a polynomial of degree $n-1$, it follows from Vieta's polynomial formulas (see for example [Vi]) that

$$
\operatorname{adj}(\mu I-A)=\left(A-\mu_{2} I\right) \cdots\left(A-\mu_{n} I\right) .
$$

Since $p_{A}^{\prime}(\mu)=\left(\mu-\mu_{2}\right) \cdots\left(\mu-\mu_{n}\right)$ this gives the desired result.

2.3. Comparison between the non-weighted case and the weighted case. When considering path counting questions on a non-weighted graph $G$, it is convenient to define its adjacency matrix. This is the square matrix $A \in M_{n}(\mathbb{R})$ indexed by the vertices of $G$, where $A_{i j}$ is set as the number of edges from vertex $i$ to vertex $j$. Note that an adjacency matrix of a strongly connected graph is irreducible, but not necessarily primitive. For primitivity of the adjacency matrix we must also assume that $G$ is aperiodic, which means that the greatest common divisor of the set of lengths of all closed paths is 1 .

Recall that the number of paths from vertex $i$ to vertex $j$ consisting of exactly $k$ edges is exactly $\left(A^{k}\right)_{i j}$. It follows that if the graph is strongly connected and aperiodic, then $A$ is primitive, and by the Perron-Frobenius theorem this number can be approximated by $P_{i j} \mu^{k}$, where $P$ is the Perron projection of $A$ described above and $\mu$ is the Perron-Frobenius eigenvalue.

It is interesting to compare the matrices $P$ and $Q$, where $Q$ is the matrix defined in the statement of Theorem 1 Due to Jacobi's formula we can write

$$
Q=\frac{\operatorname{adj}(I-M(\lambda))}{-\operatorname{tr}\left(\operatorname{adj}(I-M(\lambda)) \cdot M^{\prime}(\lambda)\right)}=\frac{\operatorname{adj}(I-M(\lambda))}{\left.\frac{d}{d s}(\operatorname{det}(I-M(s)))\right|_{s=\lambda}}
$$

and

$$
P=\frac{\operatorname{adj}\left(I-\frac{1}{\mu} A\right)}{\operatorname{tr}\left(\operatorname{adj}\left(I-\frac{1}{\mu} A\right)\right)}=\frac{\operatorname{adj}\left(I-\frac{1}{\mu} A\right)}{\left.\frac{d}{d x} \operatorname{det}\left(I-\frac{1}{x} \frac{A}{\mu}\right)\right|_{x=1}}
$$

and the resemblance is clear.

We remark that in the case of a non-weighted graph $G$ we assume that $G$ is strongly connected and aperiodic in order to guarantee convergence of $\frac{1}{\mu^{k}} A^{k}$ to $P$, otherwise the corresponding adjacency matrix need not be primitive and the Perron-Frobenius theorem may not be implied. In the case of weighted graphs we replace the assumption that $G$ is aperiodic by the assumption of incommensurability.

As an example we look at the following case: Assume all edges in $G$ are of equal length $a>0$. So $M(s)=e^{-a s} A$ where $A$ is the adjacency matrix of the underlying non-weighted graph. Obviously $G$ is not incommensurable and the assumptions of Theorem 1 do not hold, but still we can calculate $Q$.

Let $\mu$ be the Perron-Frobenius eigenvalue of $A$, then the matrix $M\left(\frac{\log \mu}{a}\right)=\frac{1}{\mu} A$ has Perron-Frobenius eigenvalue 1 , and so $\lambda=\frac{\log \mu}{a}$. Since

$$
\begin{aligned}
-\operatorname{tr}\left(\operatorname{adj}(I-M(\lambda)) \cdot M^{\prime}(\lambda)\right) & =-\operatorname{tr}\left(\operatorname{adj}\left(I-\frac{1}{\mu} A\right) \cdot \frac{-a}{\mu} A\right) \\
& =a \operatorname{tr}\left(\operatorname{adj}\left(I-\frac{1}{\mu} A\right) \cdot \frac{1}{\mu} A\right) \\
& =a \operatorname{tr}\left(\operatorname{adj}\left(I-\frac{1}{\mu} A\right)\right)
\end{aligned}
$$


we get

$$
Q=\frac{\operatorname{adj}(I-M(\lambda))}{-\operatorname{tr}\left(\operatorname{adj}(I-M) \cdot M^{\prime}(\lambda)\right)}=\frac{\operatorname{adj}\left(I-\frac{1}{\mu} A\right)}{\operatorname{atr}\left(\operatorname{adj}\left(I-\frac{1}{\mu} A\right)\right)}=\frac{1}{a} P
$$

and so if we think of a non-weighted graph as a weighted graph with edges all of length $a=1$, we get $P=Q$.

\section{The Wiener-Ikehara Theorem and the Laplace Transform}

3.1. The Wiener-Ikehara Theorem. The proofs of our main results follow from this Tauberian theorem due to Wiener and Ikehara (see chapter 8.3 in [MV]).

Theorem. Let $f(x)$ be a non-negative and monotone function on $[0, \infty)$. Suppose that the Laplace transform of $f(x)$, given by

$$
F(s):=\mathcal{L}\{f(x)\}(s)=\int_{0}^{\infty} f(x) e^{-x s} d x,
$$

converges for all $s$ with $\operatorname{Re}(s)>\lambda$, and that there exists $c \in \mathbb{R}$ for which the function

$$
F(s)-\frac{c}{s-\lambda}
$$

extends to a continuous function in the closed half-plane $\operatorname{Re}(s) \geq \lambda$. Then

$$
f(x)=c e^{\lambda x}+o\left(e^{\lambda x}\right), \quad x \rightarrow \infty .
$$

3.2. The Laplace Transform of the counting and probability functions. Denote by $\Gamma(i, j)$ the set of paths originating at vertex $i \in \mathcal{V}$ and terminating at vertex $j \in \mathcal{V}$, and by $p(\gamma)$ the product of probabilities of the edges which define the path $\gamma$.

Let $A_{i, j}(x)$ denote the number of paths originating at vertex $i$ and terminating at vertex $j$ of length at most $x$. Then

$$
A_{i, j}(x)=\sum_{\gamma \in \Gamma(i, j)} \chi_{[l(\gamma), \infty)}(x)=\sum_{k=0}^{\infty} \sum_{\substack{\gamma \in \Gamma(i, j) \\ \text { with } k \text { edges }}} \chi_{[l(\gamma), \infty)}(x)
$$

where $\chi_{A}$ is the characteristic function of the set $A \subset \mathbb{R}$. The Laplace transform is

$$
\mathcal{L}\left\{A_{i, j}(x)\right\}(s)=\sum_{k=0}^{\infty} \sum_{\substack{\gamma \in \Gamma(i, j) \\ \text { with } k \text { edges }}} \frac{1}{s} e^{-l(\gamma) s}=\frac{1}{s}\left(\sum_{k=0}^{\infty} M^{k}(s)\right)_{i, j} .
$$

Let $\alpha$ be an edge originating at vertex $j$. Denote by $B_{i, \alpha}(x)$ the number of paths of length exactly $x$ from vertex $i$ to a point on the edge $\alpha$. Then

$$
B_{i, \alpha}(x)=\sum_{\gamma \in \Gamma(i, j)} \chi_{[l(\gamma), l(\gamma)+l(\alpha))}(x)=\sum_{k=0}^{\infty} \sum_{\begin{array}{c}
\gamma \in \Gamma(i, j) \\
\text { with } k \text { edges }
\end{array}} \chi_{[l(\gamma), l(\gamma)+l(\alpha))}(x) .
$$

The Laplace transform is

$$
\mathcal{L}\left\{B_{i, \alpha}(x)\right\}(s)=\sum_{k=0}^{\infty} \sum_{\substack{\gamma \in \Gamma(i, j) \\ \text { with } k \text { edges }}} \frac{1-e^{-l(\alpha) s}}{s} e^{-l(\gamma) s}=\frac{1-e^{-l(\alpha) s}}{s}\left(\sum_{k=0}^{\infty} M^{k}(s)\right)_{i, j} .
$$

Denote by $D_{i, \alpha}(T)$ the probability that a walker leaving $i$ at time zero and moving along the graph at speed 1 , would at time $T$ be on the edge $\alpha$ which originates at vertex $j$. Then

$$
D_{i, \alpha}(T)=\sum_{\gamma \in \Gamma(i, j)} p(\gamma) p_{i j} \chi_{[l(\gamma), l(\gamma)+l(\alpha))}(T)=p_{i j} \sum_{k=0}^{\infty} \sum_{\substack{\gamma \in \Gamma(i, j) \\ \text { with } k \text { edges }}} p(\gamma) \chi_{[l(\gamma), l(\gamma)+l(\alpha))}(T)
$$


The Laplace transform is

$$
\mathcal{L}\left\{D_{i, \alpha}(T)\right\}(s)=\sum_{k=0}^{\infty} \sum_{\substack{\gamma \in \Gamma(i, j) \\ \text { with } k \text { edges }}} p_{j \alpha} \frac{1-e^{-l(\alpha) s}}{s} p(\gamma) e^{-l(\gamma) s}=p_{j \alpha} \frac{1-e^{-l(\alpha) s}}{s}\left(\sum_{k=0}^{\infty} N^{k}(s)\right)_{i, j} .
$$

It will be shown that the sums $\sum_{k=0}^{\infty} M^{k}(s)$ and $\sum_{k=0}^{\infty} N^{k}(s)$ converge absolutely for suitable values of $s$, and so we can change the order of summation and integration as implied in the calculations above.

We will show that the constant $\lambda$ as described in the statement of the Theorems 1 and 2 exists, and that these Laplace transforms satisfy the conditions of the Wiener-Ikehara theorem with a simple pole at $s=\lambda$.

\section{Proof of main Results}

Although some of the following results can be found in the literature, we include the full details for the sake of clarity.

Lemma 2. The matrix elements of powers of $M(s)$ for $s=\sigma+\mathbf{i}$ (respectively $N(s)$ ) are bounded in absolute value by the corresponding matrix elements of powers of $M(\sigma)$ (respectively $N(\sigma)$ ).

Proof. Indeed for every $k \in \mathbb{N}$

$$
\begin{aligned}
\left|\left(M^{k}(s)\right)_{i j}\right| & =\left|\sum_{i_{1}, . ., i_{k-1}} M_{i, i_{1}}(s) \cdots M_{i_{k-1}, j}(s)\right| \\
& \leq \sum_{i_{1}, . ., i_{k-1}}\left|M_{i_{i} i_{1}}(s)\right| \cdots\left|M_{i_{k-1}, j}(s)\right| \\
& \leq \sum_{i_{1}, . ., i_{k-1}} M_{i, i_{1}}(\sigma) \cdots M_{i_{k-1}, j}(\sigma)=\left(M^{k}(\sigma)\right)_{i j}
\end{aligned}
$$

and similarly for $N$, as required.

Remark 3. This lemma is contained in a result due to Wielandt which can be found in [Ga].

For $\sigma \in \mathbb{R}$ the matrices $M(\sigma)$ and $N(\sigma)$ are real, non-negative and irreducible (because the graph $G$ is strongly connected), and so by Perron-Frobenius there exists a dominant real eigenvalue $\mu(\sigma)$ of multiplicity 1 corresponding to a positive eigenvector $v(\sigma)$.

Lemma 3. Let $M(\sigma)$ be as above. Then there exists $\lambda>0$ such that $\mu(\lambda)=1$ and for every $\sigma>\lambda$ the corresponding dominant eigenvalue satisfies $\mu(\sigma)<1$.

Proof. For all $\sigma \in \mathbb{R}$ there exists $\mu(\sigma)$ which by Perron-Frobenius is a simple eigenvalue of $M(\sigma)$. Let $v(\sigma)$ and $u(\sigma)$ be right and left positive eigenvectors, then since $M(\sigma)$ is differentiable, then by Theorem 6.3.12 in [HJ] $\mu(\sigma)$ is differentiable and the following formula holds

$$
\frac{d}{d \sigma} \mu(\sigma)=\frac{u^{T}(\sigma) M^{\prime}(\sigma) v(\sigma)}{u^{T}(\sigma) v(\sigma)}
$$

Since the eigenvectors are positive, and the entry-wise derivative of $M$ is non-positive, we deduce that

$$
\frac{d}{d \sigma} \mu(\sigma)<0
$$

and in particular $\mu$ is monotone decreasing. Recall that $\mu(0)$ is the largest eigenvalue of the adjacency matrix $M(0)$ of the strongly connected and incommensurable graph $G$ and so $\mu(0)>1$. Moreover, since all elements of $M(\sigma)$ tend to zero as $\sigma$ tends to infinity, so does the Perron-Frobenius eigenvalue. Therefore there exists a finite $\lambda>0$ for which $\mu(\lambda)=1$ and $\mu(\sigma)<1$ for all $\sigma>\lambda$.

Lemma 4. Let $N(\sigma)$ be as above. Then there exists $\lambda \leq 0$ such that $\mu(\lambda)=1$ and for every $\sigma>\lambda$ the corresponding dominant eigenvalue satisfies $\mu(\sigma)<1$. 
Proof. The proof is similar to the discussion about $M(\sigma)$, only here we must show that $\mu(0) \leq 1$ to verify that the value of $\lambda$ for which $\mu(\lambda)=1$ is negative. This follows from our assumption that the sum of the probabilities of edges originating at a given vertex is less or equal to 1 , and so the sum of the entries of any row in $N(0)$ is bounded by 1 , therefore $\mu(0)$ is bounded by 1 (see Wielandt's proof of Perron-Frobenius theorem which appears in [Ga] $)$.

Remark 4. Clearly $\mu(0)=1$ if and only if the sum of all probabilities for edges originating at vertex $i$ is 1 , for all $i$. In other words $\lambda=0$ if and only if $N(0)$ is a right stochastic matrix, that is all its rows sum up to 1 .

The following Lemmas are stated for $M$, but analogous statements and their proofs apply for $N$.

Lemma 5. Let $\lambda \in \mathbb{R}$ be as in Lemma 3. Then $\sum_{k=0}^{\infty} M^{k}(\sigma)$ converges for all $s=\sigma+\mathbf{i} t$ with $\sigma>\lambda$, and in this case

$$
\sum_{k=0}^{\infty} M^{k}(s)=(I-M(s))^{-1}=\frac{\operatorname{adj}(I-M(s))}{\operatorname{det}(I-M(s))}
$$

and so the Laplace transforms of the counting functions described above are analytic in the half plane $\sigma>\lambda$.

Proof. The Lemma follows because for $\sigma>\lambda$, as in Lemma 3, the geometric sum $\sum_{k=0}^{\infty} M^{k}(\sigma)$ converges, and by Lemma 2 so does $\sum_{k=0}^{\infty} M^{k}(s)$.

Plugging the previous statement in the expressions derived in the previous section for the Laplace transforms of the counting functions we study, we conclude the following corollary.

Corollary 4. Let $A_{i, j}(x), B_{i, \alpha}(x)$ and $D_{i, \alpha}(T)$ be the counting functions defined in subsection 3.2. The associated Laplace transforms are given by

$$
\begin{aligned}
\mathcal{L}\left\{A_{i, j}(x)\right\}(s) & =\frac{1}{s} \cdot \frac{(\operatorname{adj}(I-M(s)))_{i j}}{\operatorname{det}(I-M(s))}, \\
\mathcal{L}\left\{B_{i, \alpha}(x)\right\}(s) & =\frac{1-e^{-l(\alpha) s}}{s} \cdot \frac{(\operatorname{adj}(I-M(s)))_{i j}}{\operatorname{det}(I-M(s))}, \\
\mathcal{L}\left\{D_{i, \alpha}(T)\right\}(s) & =p_{j \alpha} \frac{1-e^{-l(\alpha) s}}{s} \cdot \frac{(\operatorname{adj}(I-N(s)))_{i j}}{\operatorname{det}(I-N(s))},
\end{aligned}
$$

where $\frac{1-e^{-l(\alpha) s}}{s}$ is an entire function with value $l(\alpha)$ at $s=0$.

Lemma 6. The matrix adj $(I-M(\lambda))$ has positive entries.

Proof. Recall that $\mu(\lambda)=1$, and so by Lemma 1 there exist positive vectors $v, u$ such that

$$
\frac{\operatorname{adj}(I-M(\lambda))}{\operatorname{tr}(\operatorname{adj}(I-M(\lambda)))}=\frac{v u^{T}}{u^{T} v}
$$

It follows that all the entries of $\operatorname{adj}(I-M(\lambda))$ are non-zero and have the same sign $\operatorname{as} \operatorname{tr}(\operatorname{adj}(I-M(\lambda)))$, which is $\left.\frac{d}{d x} p_{M_{G}\left(\lambda_{G}\right)}(x)\right|_{x=1}$ by Jacobi's formula. But 1 is a simple root of the characteristic polynomial and is the largest one, and therefore its derivative at $x=1$ is positive.

Lemma 7. The Laplace transforms of the graph counting functions $A_{i, j}(x)$ and $B_{i, \alpha}(x)$ have a simple pole at $\lambda$.

Proof. The point $s=\lambda$ is a singular point of the Laplace transforms, because by the previous lemma the numerator $(\operatorname{adj}(I-M(\lambda)))_{i j}$ is non-zero while the denominator has a zero at $\lambda$. It is thus enough to show that the zero of $\operatorname{det}(I-M(s))$ at $\lambda$ is a simple one. For $\sigma \in \mathbb{R}$, the characteristic polynomial of $M(\sigma)$ is given by

$$
p_{M(\sigma)}(x)=\operatorname{det}(x I-M(\sigma))=(x-\mu(\sigma))\left(x-\mu_{2}(\sigma)\right) \cdots\left(x-\mu_{n}(\sigma)\right),
$$

where $\mu(\sigma)$ is the Perron-Frobenius eigenvalue of $M(\sigma)$. Therefore $\mu(\lambda)=1$, and $\mu_{j}(\sigma) \neq 1$ for $j \geq 2$ in a small neighborhood of $\lambda$, and

$$
\operatorname{det}(I-M(\sigma))=(1-\mu(\sigma)) \cdots\left(1-\mu_{n}(\sigma)\right) .
$$

It follows from equation 4.1 that the function $(1-\mu(\sigma))$ has a simple zero at $\lambda$, and the same holds for the function $\operatorname{det}(I-M(\sigma))$ and therefore also for $\operatorname{det}(I-M(s))$. 
Lemma 8. For all $t \neq 0$,

$$
\operatorname{det}(I-M(\lambda+\mathbf{i} t)) \neq 0,
$$

that is the Laplace transforms have no other poles on the line $\operatorname{Re}(s)=\lambda$ than at $s=\lambda$ itself.

Proof. Say that $G$ has the single-edge property if for every pair of vertices $i, j$ in $G$ there is at most one edge from vertex $i$ to vertex $j$. Given a graph $G$, we define a graph $\widetilde{G}$ by adding a new vertex in the middle of every edge in $G$. There is a natural one to one map between paths in $G$ and paths in $\widetilde{G}$ which originate and terminate in the original set of vertices, and clearly $\widetilde{G}$ has the single-edge property. Therefore, there is no loss of generality assuming that this property holds for $G$.

Put $M_{i j}=(M(\lambda))_{i j}$. Since $G$ has the single-edge property, the entries of the matrix $M(\lambda)$ are either $M_{i j}=e^{-\lambda \cdot l(\alpha)}$ if there is an edge $\alpha \in \mathcal{E}$ connecting the vertex $i$ to the vertex $j$, or $M_{i j}=0$ if there is no such edge. Let $v=\left(v_{1}, \ldots, v_{n}\right)$ be a positive eigenvector of $M(\lambda)$ corresponding to the eigenvalue $\mu(\lambda)=1$ and define $D$ to be the following invertible matrix

$$
D=\operatorname{diag}\left(v_{1}, \ldots, v_{n}\right), \quad D^{-1}=\operatorname{diag}\left(\frac{1}{v_{1}}, \ldots, \frac{1}{v_{n}}\right) .
$$

The matrix given by $S=D^{-1} M D$ is a non-negative and right stochastic matrix. Indeed, since $S_{i j}=$ $M_{i j} v_{j} / v_{i}$, it is clear that $S_{i j} \geq 0$ and that the sum of the elements of the $i$ th row is

$$
\sum_{j=1}^{n} S_{i j}=\sum_{j=1}^{n} M_{i j} \frac{v_{j}}{v_{i}}=\frac{1}{v_{i}} \sum_{j=1}^{n} M_{i j} v_{j}=\frac{v_{i}}{v_{i}}=1 .
$$

Let $S(s)$ be the matrix with coefficients from $S$ raised to the power of $s$, that is

$$
(S(s))_{i j}=\left(S_{i j}\right)^{s}=\left(M_{i j}\right)^{s}\left(\frac{v_{j}}{v_{i}}\right)^{s} .
$$

Notice that

$$
S(s)=\left(D^{s}\right)^{-1} M(\lambda s) D^{s},
$$

that is $M(\lambda s)$ and $S(s)$ are similar, and in particular they have the same characteristic polynomial $p_{S(s)}(x)=p_{M(\lambda s)}(x)$. Recalling the definition of the characteristic polynomial and plugging $x=1$ we see that

$$
\operatorname{det}(I-M(\lambda s))=\operatorname{det}(I-S(s))
$$

and so it is enough to show that $\operatorname{det}(I-S(1+\mathbf{i} t)) \neq 0$ for all $t \neq 0$.

The following argument is due to Parry (see $[\mathrm{Pa}])$. Assume $\operatorname{det}(I-S(1+\mathbf{i} t))=0$ for some $t \neq 0$. So there exists a non-zero vector $u=\left(u_{1}, \ldots, u_{n}\right)$ for which

$$
S(1+\mathbf{i} t) u=u,
$$

that is for all $i$

$$
\sum_{j=1}^{n} S_{i j}^{1+\mathbf{i} t} u_{j}=\sum_{j=1}^{n} S_{i j} S_{i j}^{\mathbf{i} t} u_{j}=u_{i}
$$

By the triangle inequality, for all $i$

$$
\left|u_{i}\right| \leq \sum_{j=1}^{n}\left|S_{i j}^{1+\mathbf{i} t} u_{j}\right|=\sum_{j=1}^{n} S_{i j}\left|S_{i j}^{\mathbf{i} t}\right|\left|u_{j}\right|=\sum_{j=1}^{n} S_{i j}\left|u_{j}\right|,
$$

and together with the equality

$$
\sum_{j=1}^{n} S_{i j}=1
$$

this implies that

$$
\left|u_{1}\right|=\ldots=\left|u_{n}\right| \text {. }
$$

Assume $\left|u_{j}\right|=r>0$ for all $j$, and notice that this means $S_{i j}^{\mathbf{i t}} u_{j}$ are points on a circle of radius $r$. We have therefore that every $u_{i}$, which is itself a point on the circle of radius $r$, is a convex combination (that is, a linear combination with positive coefficients all adding up to 1) of points on that same circle. This is only possible of course if $S_{i j}^{\mathrm{it}} u_{j}=u_{i}$ for all $j$ such that $S_{i j} \neq 0$. 
Now, for any closed orbit on the graph, let $\alpha_{1}=\left(k_{1}, k_{2}\right), \ldots, \alpha_{m}=\left(k_{m}, k_{1}\right)$ denote the corresponding sequence of edges. We get

$$
\left(S_{k_{1} k_{2}}^{\mathrm{i} t} u_{k_{2}}\right)\left(S_{k_{2} k_{3}}^{\mathrm{i} t} u_{k_{3}}\right) \ldots\left(S_{k_{m-1} k_{m}}^{\mathrm{i} t} u_{k_{m}}\right)\left(S_{k_{m} k_{1}}^{\mathrm{i} t} u_{k_{1}}\right)=u_{k_{1}} u_{k_{2}} \ldots u_{k_{m-1}} u_{k_{m}}
$$

and so

which gives

$$
S_{k_{1} k_{2}}^{\mathrm{it}} \ldots S_{k_{m} k_{1}}^{\mathrm{i} t}=\left(S_{k_{1} k_{2}} \ldots S_{k_{m} k_{1}}\right)^{\mathrm{i} t}=1
$$

$$
\left(M_{k_{1} k_{2}} \ldots M_{k_{m} k_{1}}\right)^{\mathrm{i} t}=1
$$

But

$$
M_{k_{1} k_{2} \ldots M_{k_{m}} k_{1}}=e^{-\left(l\left(\alpha_{1}\right)+\ldots+l\left(\alpha_{m}\right)\right)}
$$

and so there exists some $l \in \mathbb{Z}$ for which

$$
t=\frac{2 \pi l}{l\left(\alpha_{1}\right)+\ldots+l\left(\alpha_{m}\right)} .
$$

This hold for every closed orbit on the graph, which is a contradiction to our irrationality assumptions on the lengths of the closed orbits on our incommensurable graph $G$.

Remark 5 . When proving the analogous Lemma for the case of the matrix $N$, simply assign the probability 1 to all edges of $\widetilde{G}$ originating at vertices of $\widetilde{G}$ which are not in $G$.

Remark 6 . There is another construction of a graph $\widehat{G}$ associated to $G$ which preserves its structure and has the single-edge property. Let $i \in \mathcal{V}$ be a vertex in $G$ and assume that there are $k_{i}$ distinct edges which terminate at $i$ and $l_{i}$ distinct edges which originate at $i$ as shown in Figure 2

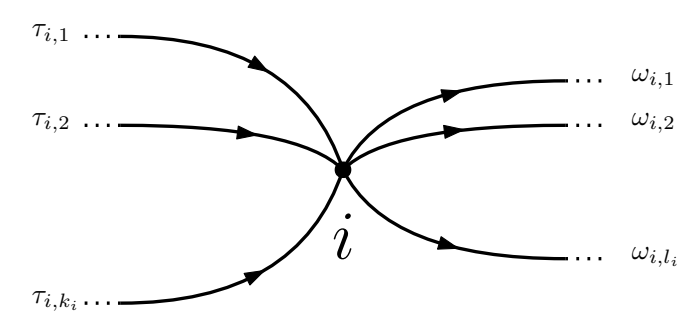

Figure 2. Vertex $i \in \mathcal{V}$ and the edges which terminate and originate at $i$ in $G$

Define $k_{i} \cdot l_{i}$ associated vertices in $\widehat{G}$, indexed by

$$
\begin{array}{ccc}
\tau_{i 1}-i-\omega_{i 1}, & \ldots & \tau_{i k_{i}}-i-\omega_{i 1} \\
\vdots & & \vdots \\
\tau_{i 1}-i-\omega_{i l_{i}}, & \ldots & \tau_{i k_{i}}-i-\omega_{i l_{i}}
\end{array}
$$

and repeat this procedure for all vertices in $G$. Define $\widehat{G}$ to contain a directed edge from $\widehat{v}_{1}=\tau_{i s}-i-\omega_{i t}$ to $\widehat{v}_{2}=\tau_{j u}-j-\omega_{j v}$ if and only if $\omega_{i t}=\tau_{j u}$. If there is such an edge, and if $\alpha$ is the edge in $G$ for which $\alpha=\omega_{i t}=\tau_{j u}$, then the edge between $\widehat{v}_{1}$ and $\widehat{v}_{2}$ is labeled by $\alpha$.

It can be shown that for every path $p$ in $G$ which originates in vertex $i$ and terminates in vertex $j$, there are exactly $k_{i} \cdot l_{j}$ distinct paths in $\widehat{G}$ which are copies of $p$ in the sense of the edges they consist of. It follows that functions counting paths in $G$ which concern vertices $i$ and $j$ differ by a multiplicative constant from the associated functions on $\widehat{G}$, and the same holds for their Laplace transforms. As a result, the position of the poles of the Laplace transforms is not changed.

Lemma 9. The residue of the function $\frac{\operatorname{adj}(I-M(s))_{i j}}{\operatorname{det}(I-M(s))}$ at $s=\lambda$ is

$$
Q_{i j}=\frac{(\operatorname{adj}(I-M(\lambda)))_{i j}}{-\operatorname{tr}\left(\operatorname{adj}(I-M(\lambda)) \cdot M^{\prime}(\lambda)\right)}
$$


Proof. We have seen that the function has a simple pole at $s=\lambda$, and so the residue at $s=\lambda$ is

$$
\frac{(\operatorname{adj}(I-M(\lambda)))_{i j}}{\left.\frac{d}{d s}(\operatorname{det}(I-M(s)))\right|_{s=\lambda}} .
$$

Finally, use Jacobi's formula to obtain

$$
\begin{aligned}
\frac{d}{d s}(\operatorname{det}(I-M(s))) & =\operatorname{tr}\left(\operatorname{adj}(I-M(\lambda)) \cdot(I-M(s))^{\prime}(\lambda)\right) \\
& =-\operatorname{tr}\left(\operatorname{adj}(I-M(\lambda)) \cdot M^{\prime}(\lambda)\right) .
\end{aligned}
$$

Combining the above we get the desired formula for the residue at hand.

4.1. Proof of Theorems $\mathbf{1}$ and 2, We show how to conclude the main statements of this paper from the Lemmas proven above.

Proof of Theorem 1. Let $G$ be a strongly connected incommensurable graph and let $M$ be the graph matrix function of $G$. For $\sigma \in \mathbb{R}$ the matrix $M(\sigma)$ is real and due to Perron-Frobenius there exists a dominant real eigenvalue $\mu(\sigma)$ of multiplicity 1. By Lemma 3 there exists $\lambda>0$ such that $\mu(\lambda)=1$ and for every $\sigma>\lambda$ the corresponding dominant eigenvalue satisfies $\mu(\sigma)<1$. By Lemma 2 the series $\sum_{k=0}^{\infty} M^{k}(s)$ converges for all $s=\sigma+\mathbf{i} t$ with $\sigma>\lambda$ to $\frac{\operatorname{adj}(I-M(s))}{\operatorname{det}(I-M(s))}$ and so by Corollary 4

$$
\mathcal{L}\left\{A_{i, j}(x)\right\}(s)=\frac{1}{s} \cdot \frac{(\operatorname{adj}(I-M(s)))_{i j}}{\operatorname{det}(I-M(s))} .
$$

By Lemma 7 this $\mathcal{L}\left\{A_{i, j}(x)\right\}(s)$ has a simple pole at $s=\lambda$ and by Lemma 8 there are no other poles on the line $\operatorname{Re}(s)=\lambda$. By Lemma 9 the residue of $\mathcal{L}\left\{A_{i, j}(x)\right\}(s)$ at $s=\lambda$ is

$$
\frac{1}{\lambda} \frac{(\operatorname{adj}(I-M(\lambda)))_{i j}}{-\operatorname{tr}\left(\operatorname{adj}(I-M(\lambda)) \cdot M^{\prime}(\lambda)\right)}=\frac{1}{\lambda} Q_{i j} .
$$

Therefore applying the Wiener-Ikehara Theorem yields statement $(i)$, namely that the number of paths from $i \in \mathcal{V}$ to $j \in \mathcal{V}$ of length at most $x$ grows as

$$
\frac{1}{\lambda} Q_{i j} e^{\lambda x}+o\left(e^{\lambda x}\right), \quad x \rightarrow \infty .
$$

Replacing $A_{i, j}(x)$ with $B_{i, j}(x)$ and repeating these steps yields statement $(i i)$, namely that the number of paths of length exactly $x$ from some vertex $i$ to a point on the edge $\alpha$ grows a

$$
\frac{1-e^{-l(\alpha) \lambda}}{\lambda} Q_{i j} e^{\lambda x}+o\left(e^{\lambda x}\right), \quad x \rightarrow \infty .
$$

The proof of Theorem 2 is analogous to the proof of Theorem 1 described above. Lemma 3 is replaced by Lemma 4 and as mentioned above, Lemmas analogous to Lemmas $5 \sqrt{9}$ hold when replacing $M$ with the graph probability matrix function $N$.

\section{Applications}

5.1. Summation over regions of Pascal triangle. The well known triangular array of binomial coefficients contains many patterns of numbers and properties of combinatorial interest. It is a straightforward observation that summation of the binomial coefficients in the triangle $O B A$ of sides $O A=\frac{x}{a}$ and $O B=\frac{x}{b}$ (see Figure 3 is equivalent to counting paths of length at most $x$ in a graph with a single vertex and two loops of lengths $a$ and $b$.

This easily generalizes to Pascal pyramids of higher dimension and weighted graphs with a singe vertex and several loops, and it would be interesting to understand the full correspondence between weighted graphs and regions in Pascal pyramids. 

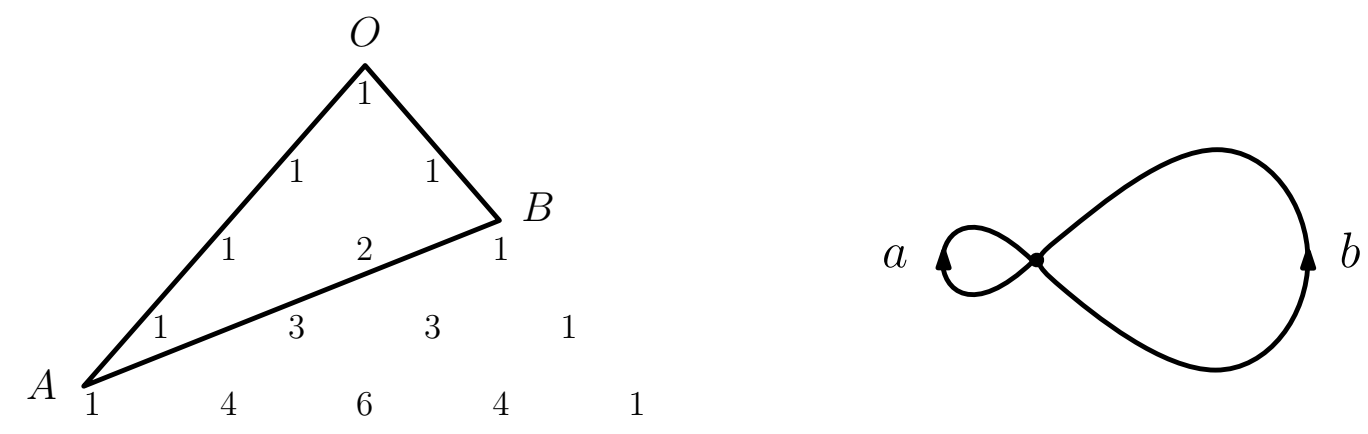

Figure 3. A region in Pascal's triangle and the associated graph.

5.2. Multiscale Substitution Schemes. A tile $T$ in $\mathbb{R}^{d}$ is a Lebegue measurable bounded set with positive measure and boundary of measure zero. Consider a finite set of tiles $\mathcal{F}=\left\{\mathcal{T}_{1}, \ldots, \mathcal{T}_{n}\right\}$ in $\mathbb{R}^{d}$ which we call prototiles, and assume for simplicity $\operatorname{vol} \mathcal{T}_{i}=1$. A tile $T$ is said to be of type $i$ if it maps to $\mathcal{T}_{i}$ by a similarity map. A multiscale substitution scheme on $\mathcal{F}$ is a set of substitution rules on elements of $\mathcal{F}$ prescribing a tiling of each prototile by finitely many rescaled copies of tiles of types appearing in $\mathcal{F}$.

A multiscale substitution scheme can be modeled using a directed weighted graph $G$ with a vertex set indexed by elements of $\mathcal{F}$ and an edge set defined by the substitution rule: if the tiling of $\mathcal{T}_{i}$ includes a tile of type $j$, that is a copy of $\alpha \mathcal{T}_{j}$ with $0<\alpha<1$, then $G$ admits a directed edge of length $a=-\log \alpha$ connecting vertex $i$ to $j$. A multiscale substitution scheme is called irreducible if $G$ is strongly connected, and incommensurable if $G$ is incommensurable. An example of an incommensurable multiscale substitution scheme on a single prototile with scales $\alpha_{1}=\frac{1}{3}$ and $\alpha_{2}=\frac{2}{3}$ is shown in Figure 4
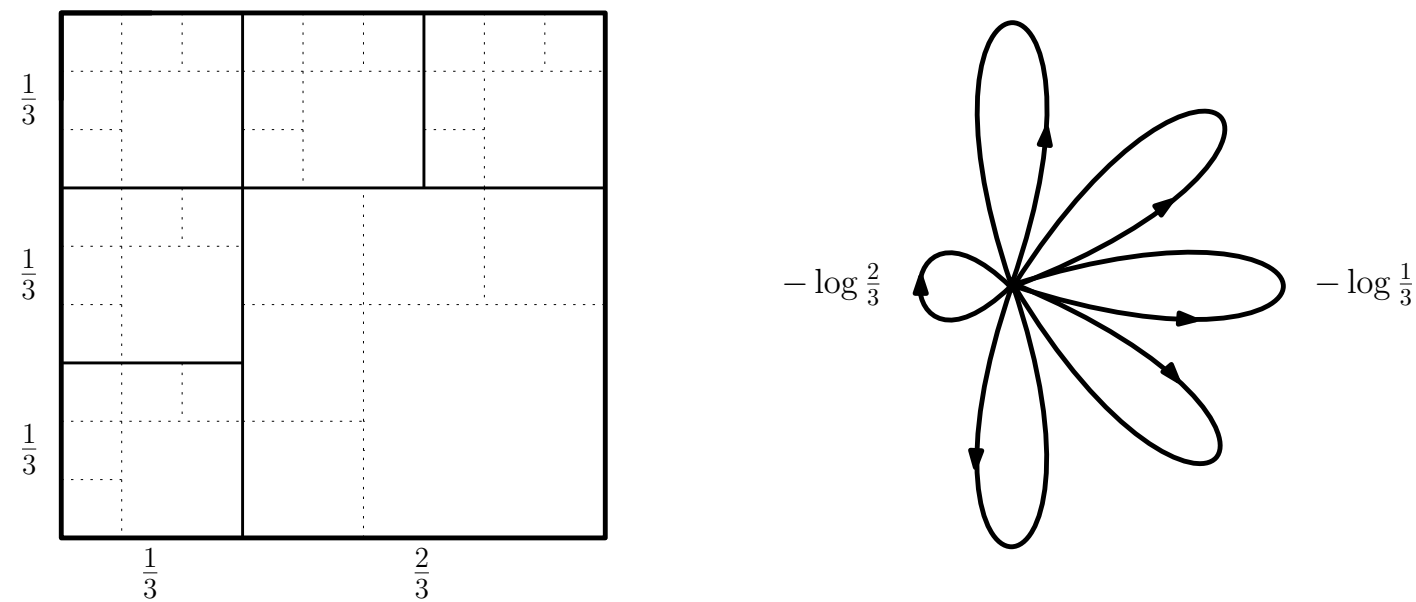

FigURE 4. A multiscale substitution scheme and the associated graph.

Observe that if $G$ is a graph associated with a $d$ dimensional multiscale substitution scheme, then $\lambda=d$ and the Perron-Frobenius eigenvector of $M(d)$ corresponding to $\mu=1$ can be chosen to be $v=(1, \ldots, 1)$. These properties enable us to address questions concerning the geometrical objects described bellow.

Kakutani Splitting Procedure. Consider the unit interval $I=[0,1]$ and some $\alpha \in(0,1)$. Kakutani introduced the following splitting procedure which generates a sequence of partitions of $I$ which is known as the $\alpha$-Kakutani's sequence of partitions (see $\mathrm{Ka}$ ). Begin with $\pi_{0}=I$ the trivial partition of $I$, and define $\pi_{1}$ to be the partition of $I$ one gets after splitting $I$ into two intervals of lengths $\alpha$ and $1-\alpha$. Assume that the partition $\pi_{n}$ is defined, then $\pi_{n+1}$ is the partition of $I$ one gets from $\pi_{n}$ after splitting the interval of maximal length in $\pi_{n}$ into two parts, proportional to $\alpha$ and $1-\alpha$. 
For example, the first few $\alpha$-Kakutani partitions of the unit interval with $\alpha=\frac{1}{3}$ are shown in Figure 5 together with the associated graph. The dashed lines represent intervals of maximal length in each partition.
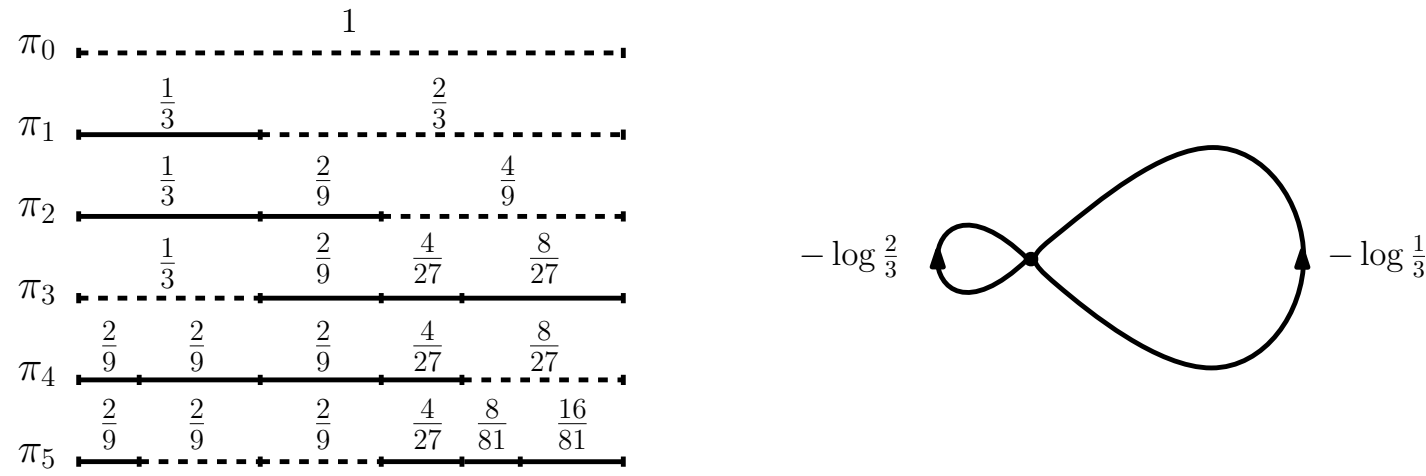

Figure 5. The $\frac{1}{3}$-Kakutani sequence of partitions and the associated graph.

We say that a sequence $\pi_{n}$ of partitions of $I$ is uniformly distributed if for any continuous function $f$ on $I$ we have

$$
\lim _{n \rightarrow \infty} \frac{1}{k(n)} \sum_{i=1}^{k(n)} f\left(t_{i}^{(n)}\right)=\int_{I} f(t) d t
$$

where $k(n)$ is the number of intervals in the partition $\pi_{n}$, and $t_{i}^{(n)}$ is the right endpoint of the $i$ th interval in the partition $\pi_{n}$. The following result is due to Kakutani.

Theorem. For any $\alpha \in(0,1)$, the $\alpha$-Kakutani sequence of partitions of I is uniformly distributed.

Kakutani's splitting procedure is generalized in various ways, see for example [CV] and [Vo. An additional generalization comes from multiscale substitution schemes, where we begin with an initial tile $\mathcal{T}_{1}$, and define a sequence of partitions of $\mathcal{T}_{1}$ using the substitution rule applied at each stage to tiles of maximal volume. In fact, the example given above of Kakutani's original procedure, can be considered as generated by a multiscale substitution scheme in $\mathbb{R}^{1}$ with $\mathcal{F}=\{I\}$ the unit interval and $\alpha_{1}=\frac{1}{3}$ and $\alpha_{2}=\frac{2}{3}$.

Using Theorem 1] it is shown in [SmY] that Kakutani splitting procedures which correspond to incommensurable multiscale substitution schemes are uniformly distributed.

Multiscale Substitution Tilings of Euclidean Spaces. A multiscale substitution scheme in $\mathbb{R}^{d}$ can be used to generate a tiling of the entire space. We define a sequence of tilings of finite regions of $\mathbb{R}^{d}$ which depends on a continuous time parameter $t$ in the following way: At $t=0$ apply the substitution rule on an initial tile $\mathcal{T}_{1}$, and inflate the resulting patch of tiles at a constant speed. Any tile which reaches volume 1 is then substituted as dictated by the multiscale substitution rule, and so on. An appropriate compact topology defined on closed subsets of the space allows us to take limits of sequences of these partial tilings, and these limits define tilings of $\mathbb{R}^{d}$. The generalization of the pinwheel tiling which is presented in $\mathrm{Sa}$ ] can be regarded as a multiscale substitution tiling.

Although there is no uniqueness in the construction of tilings using multiscale substitutions, all tilings defined this way share various properties which can be analyzed using the multiscale substitution scheme itself and the underlying weighted graph. For example, tilings which are associated with incommensurable multiscale substitution schemes are of infinite local complexity, unlike classical substitution tilings or tilings defined using cut-and-project constructions (for more on tilings and mathematical models of quasicrystals see [BG]). Our Theorem 1 may be used to study various statistics of these tilings, see [SyS for more details, and So1 and So2 for relevant results concerning classical substitution tilings.

5.3. Physics Applications. The propagation of radiation pulses through networks of wave-guides requires for its study the full theory of wave dynamics, where interference effects play an important role (see [ScS] and references therein). However, under certain circumstances the interference effects can be neglected, which opens the possibility to study this system within a classical dynamics setting: The 
network is modeled by a metric, directed graph $G$, where for any directed edge $\alpha$ connecting vertex $u$ to vertex $v$, there exists a "reverse" edge $\widehat{\alpha}$ from $v$ to $u$ of the same length. The vertices correspond to the junctions in the network where wave-guides are connected. In the classical model, one considers a point mass moving at a unit speed along a directed edge $\alpha$, towards the vertex $v$. Reaching $v$, the point mass is scattered into any of the edges $\alpha^{\prime}$ which emanate from $v$ where it continues to move with unit speed. The probabilities to make the transitions from $\alpha$ to $\alpha^{\prime}$ are prescribed by the properties of the connectors in the wave-guide network.

The network is connected to the outside world through leads which are coupled to a subset of vertices $\mathcal{H}$. One of the vertices $s \in \mathcal{H}$ is connected to a radiation source which sends short pulses to the network at specified times. Another vertex $t \in \mathcal{H}$ is connected to a lead which ends with a detector where the time of arrival is measured. The radiation which is scattered to the leads escapes from the network. In the classical model, a particle is injected to the vertex $s$ at a given time, and once it scattered from $t$ to the lead, its arrival time is measured. Repeating the process one can obtain the probability distribution of the transition times. This model can be analyzed within the formalism provided by Theorem 2 .

The complete wave theory and the derivation of the corresponding classical model are provided in ScS. This paper also includes an analysis of a simple network (similar to the one shown here in Figure 3 with the lead connected at the single vertex) and the transition time distributions computed both in the wave and the classical descriptions are compared.

\section{REFERENCES}

[An] Anlage, S., 2016. Private communication.

[BG] Baake, M. and Grimm, U.,2013. Aperiodic order. Vol. 1. A mathematical invitation. Encyclopedia of Mathematics and its Applications, 149. Cambridge University Press.

[BPP] Broise-Alamichel, A., Parkkonen, J. and Paulin, F., 2016. Equidistribution and counting under equilibrium states in negatively curved spaces and graphs of groups. Applications to non-Archimedean Diophantine approximation, arXiv:1612.06717 1 .

[CT] Chernyshev, V. L. and Tolchennikov, A. A., 2011. The properties of the distribution of Gaussian packets on a spatial network, Nauka i Obrazovanie 10, 1-10

[CV] Carbone, I. and Volčič, A., 2007. Kakutani's splitting procedure in higher dimension, Rend. Ist. Matem. Univ. Trieste XXXIX, 1-8.

[FDC] Frazer, R. A., Duncan W.J. and Collar A.R., 1955. Elementary Matrices and Some Applications to Dynamics and Differential Equations, Cambridge University Press.

[Ga] Gantmacher, F.R., 1979. The theory of matrices, vol. 2, Chelsea, New York, 1959. Mathematical Reviews (MathSciNet): MR99f, 15001.

[GS] Gavish, U. and Smilansky, U., 2007. Degeneracies in the length spectra of metric graphs. Journal of Physics A: Mathematical and Theoretical, 40(33), p.10009.

[Gu] Guillope, L., 1994. Entropies et spectres, Osaka J. Math. 31, 247-289.

[HJ] Horn, R.A. and Johnson, C.R., 1985. Matrix analysis. Cambridge university press.

[Ka] Kakutani, S., 1976. A problem on equidistribution on the unit interval [0,1], Measure Theory, Oberwolfach 1975, vol. 541, pp. 369-375. Springer LNM.

[KS] Kenison, G. and Sharp, R., 2017. Orbit counting in conjugacy classes for free groups acting on trees. Journal of Topology and Analysis, 9(04), pp.631-647.

[MV] Montgomery, H.L. and Vaughan, R.C., 2006. Multiplicative number theory I: Classical theory (Vol. 97). Cambridge University Press.

[Pa] Parry, W., 1983. An analogue of the prime number theorem for closed orbits of shifts of finite type and their suspensions. Israel Journal of Mathematics, 45(1), pp.41-52.

[PP1] Parry, W. and Pollicott, M., 1983. An analogue of the prime number theorem for closed orbits of Axiom A flows. Annals of mathematics, pp.573-591.

[PP2] Parry, W. and Pollicott, M., 1990. Zeta functions and the periodic structure of hyperbolic dynamics. Asterisque 187-188.

[Sa] Sadun, L., 1998. Some generalizations of the pinwheel tiling. Discrete \& Computational Geometry, 20(1), pp.79-110.

[ScS] Schanz, H. and Smilansky U., 2017. Delay time distribution in the scattering of time narrow wave packets (II) Quantum Graphs. J. Phys. A: Math. Theor. 51, 075302.

[Se] Seneta, E., 1981. Non-negative matrices and Markov chains. Springer Series in Statistics. Springer, New York.

[SmU] Smilansky, U., 2017. Delay-time distribution in the scattering of time-narrow wave packets. (I) J. Phys. A: Math. Theor. 50, 215301.

[SmY] Smilansky Y., 2018. Uniform distribution of Kakutani partitions generated by substitution schemes, arXiv:1805.02213 [math.DS].

[SyS] Smilansky, Y. and Solomon, Y., Multiscale substitution tilings, in preparation.

[So1] Solomon, Y., 2011. Substitution tilings and separated nets with similarities to the integer lattice, Israel J. Math. $181,445-460$

[So2] Solomon, Y., 2014. A simple condition for bounded displacement. J. Math. Anal. Appl. 414, no. 1, 134-148. 
[Vi] Viète, F., 1646. "Opera Mathematica" F. van Schouten (ed.), Leiden.

[Vo] Volčič, A., 2011. A generalization of Kakutani’s splitting procedure. Ann. Mat. Pura. Appl., Vol. 190, pp. 45-54.

Avner Kiro, Raymond and Beverly Sackler School of Mathematical Sciences, Tel Aviv University, Tel Aviv 69978, ISRAEL

E-mail address: avnerefrak@mail.tau.ac.il

Yotam Smilansky, Raymond and Beverly Sackler School of Mathematical Sciences, Tel Aviv University, TEL AVIV 69978, IsRAel

E-mail address: yotam.smilansky@mail.huji.ac.il

Uzy Smilansky, Department of Physics of Complex Systems, The Weizmann Institute of Science, Rehovot 76100, ISRAEL

E-mail address: uzy.smilansky@weizmann.ac.il 